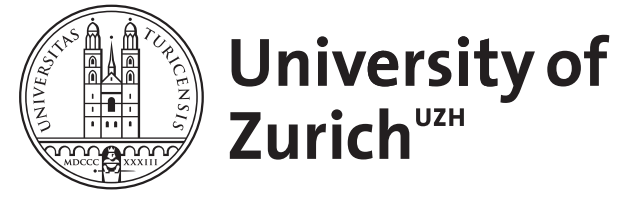
Archive

University of Zurich

University Library

Strickhofstrasse 39

CH-8057 Zurich

www.zora.uzh.ch

Year: 1996

\title{
Lokalisierung in der 1H-MR-Spektroskopie
}

Faas, H M ; Weber, O M ; Duc, C O ; Pruessmann, K P ; Meier, D ; Boesiger, P

DOI: https://doi.org/10.1515/bmte.1996.41.s1.224

Posted at the Zurich Open Repository and Archive, University of Zurich

ZORA URL: https://doi.org/10.5167/uzh-154609

Journal Article

Published Version

Originally published at:

Faas, H M; Weber, O M; Duc, C O; Pruessmann, K P; Meier, D; Boesiger, P (1996). Lokalisierung in der 1H-MR-Spektroskopie. Biomedizinische Technik. Biomedical engineering, 41(s1):224-225.

DOI: https://doi.org/10.1515/bmte.1996.41.s1.224 


\title{
Lokalisierung in der ${ }^{1} \mathrm{H}$-MR-Spektroskopie
}

\author{
H. M. Faas, O. M.Weber, C. O. Duc, K. P. Pruessmann, \\ D. Meier, P. Boesiger \\ Institut für Biomedizinische Technik und Medizinische Informatik \\ Universität und ETH Zürich, CH-8044 Zürich, Schweiz
}

\section{EINLEITUNG}

Die volumenselektive Magnetresonanzspektroskopie (MRS) ermöglicht die nichtinvasive Beobachtung von metabolischen Prozessen in vivo. Dadurch sollen Erkenntnisse über neurologische Zusammenhänge und Störungen gewonnen werden. Auf den klinischen Ganzkörpertomographen bestimmt die Pulssequenz das Messvolumen. Für die Interpretation der Messdaten ist die Kenntnis der Lokalisierungsqualität von grosser Bedeutung.

Bei der Doppelspinecho-Sequenz PRESS wird die Volumenselektion durch drei RF-Pulse mit parallel geschalteten, zueinander orthogonalen Gradienten erreicht. Da die schmalbandigen RF-Pulse keine perfekte Schichtselektion bewirken, ergibt sich im Randbereich des Messvolumens eine breite Flipwinkelverteilung, die das MRS-Signal stark beeinflussen kann. Das tatsächliche Anregungsprofil im Zielvolumen wird von der Form der RF-Pulse bestimmt. Zur Beurteilung der Lokalisierungsqualität dienen in erster Linie die Effizienz der Lokalisierung sowie die Kontamination des Signals durch Spins ausserhalb des Nominalvolumens.

Ziel dieser Untersuchung war es, für klinisch relevante Metaboliten die Güte der Lokalisierung zu bestimmen und im Fall von Substanzen mit Kopplungen die Einflüsse des Pulsprofils auf das MR-Spektrum zu betrachten. Dazu wurden numerische Simulationen und experimentelle Phantommessungen durchgeführt und die Ergebnisse verglichen. Da pathologische Erscheinungen häufig auf sehr kleine Geweberegionen beschränkt sind [1], wurde bei den Messungen insbesondere auch der Fall geringer Volumina ( $>1 \mathrm{ml}$ ) betrachtet.

\section{SIMULATION}

Ein Simulationsprogramm [4] erlaubt die Darstellung der makroskopischen Magnetisierung unter dem Einfluss der RF-Pulse und Gradienten. Die Berechnung der Magnetisierung erfolgt dabei durch die numerische Lösung der Blochgleichungen. Zunächst wurden einzelne Pulse, dann die gesamte Sequenz simuliert. Während der $90^{\circ}$-Anregungspuls eine gute Schichtdefinition aufweist, wird die Lokalisierung vor allem durch die Form der $180^{\circ}$-Refokussierpulse limitiert ( Bild I). Dadurch ergibt sich nur in eine Raumrichtung eine recht scharfe Volumenbegrenzung, hingegen resultieren deutliche Abweichungen in den durch die Refokussiergradienten festgelegten Richtungen. Die. Simulation der ganzen Sequenz zeigt, dass nur etwa $50 \%$ des Volumens
Flipwinkel im Bereich des Nominalwinkels ( $\pm 5 \%$ Abweichung) aufweisen (Bild 2).

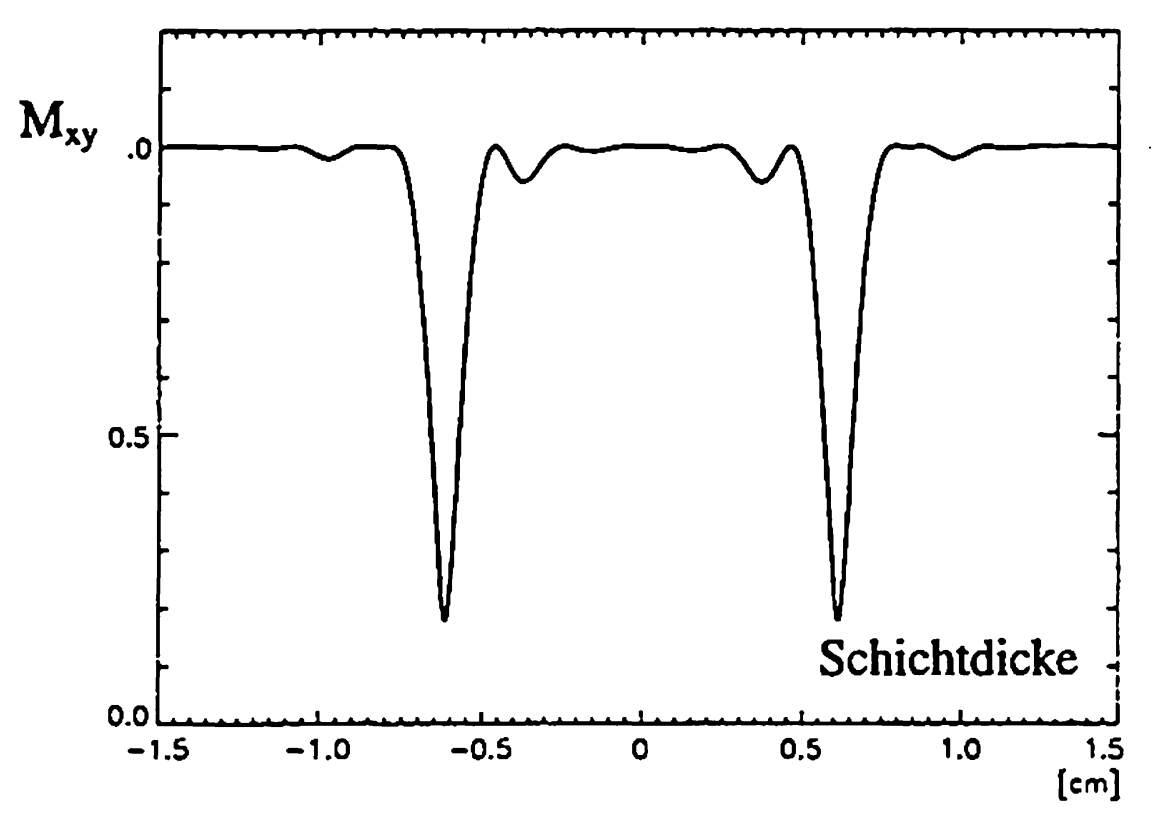

Bild 1: Betrag der transversalen Magnetisierung $M_{x \rightarrow}$ nach einem optimierten $180^{\circ}$-Puls in der Simulation.

Bei Veränderung der Schichtdicke durch Anpassen der Gradientenstärke bleibt das Verhältnis von Randbereich zu Kernzonenausdehnung konstant. Dies bedeutet, dass die Flanken des Anregungsprofils bei einer Vergrösserung des Volumens proportional flacher werden.

\section{EXPERIMENTE}

Die Experimente wurden mit einem klinischen $1.5 \mathrm{~T}$ Ganzkörpertomographen (Philips Gyroscan ACS/NT) durchgeführt. Als Sender und Empfänger diente eine Philips-Standard-Kopfspule. Zur Unterdrückung des Wassersignals wurden frequenzselektive Pulse und Spoilergradienten der PRESS-Sequenz vorgeschaltet (CHESS).

Zur experimentellen Beurteilung der Lokalisierung wurde ein Zweikomponentenphantom erstellt. Ein Kubus (15 mm Kantenlänge) wurde in einem äusseren Kompartiment mit einer Referenzlösung aufgehängt. Das Messvolumen wurde um das innere Kompartiment zentriert und in der Grösse variiert. Die wässrige Lösung im Innern des Würfels bestand aus $33.3 \mathrm{mM}$ N-Acetylaspartat (NAA) und $50 \mathrm{mM}$ Laktat bei pH 7 (zusätzlich $\mathrm{NiCl}_{2}$ zur Senkung der Relaxationszeiten). Im äusseren Volumen befanden sich als Referenzlösung $33.3 \mathrm{mM}$ Kreatin und $10 \mathrm{mM}$ Valin bei $\mathrm{pH} 7$ (ausserdem $\mathrm{NiCl}_{2}$ ). Um mögliche Suszeptibilitätsartefakte bei kleinen Volumina auszuschliessen, wurde ein sphärisches Kompartiment mit $4 \mathrm{~cm}$ Durchmesser in einem 5-Liter- 
Gefäss verwendet. So konnten die Kontamination und Effizienz der Lokalisierung bestimmt werden, indem das Messvolumen soweit verkleinert wurde, bis es mit dem Kubus gerade übereinstimmte.

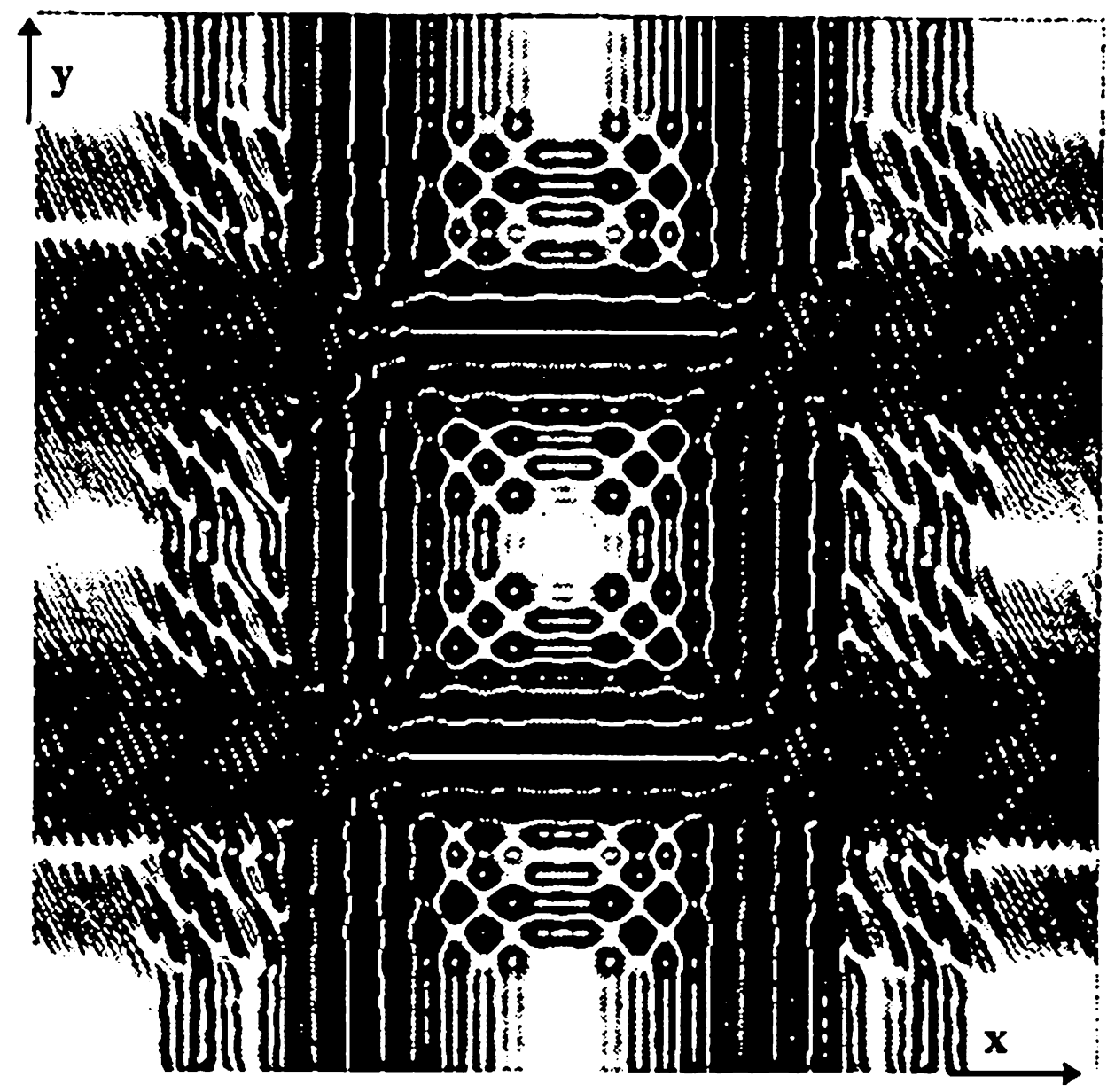

Bild 2: Betrag der transversalen Magnetisierung $M_{x \text {; }}$ nach der ganzen PRESS-Sequenz in der Simulation. Dargestellt ist die Ebene, die durch die Refokussiergradienten gewählt wird. Dabei entsprechen die hellen Bildteile den nominalen Flipwinkeln. Der Kernzone (heller Bereich in der Mitte) steht ein Randbereich (dunkler Teil) von etwa derselben Ausdehnung gegenüber.

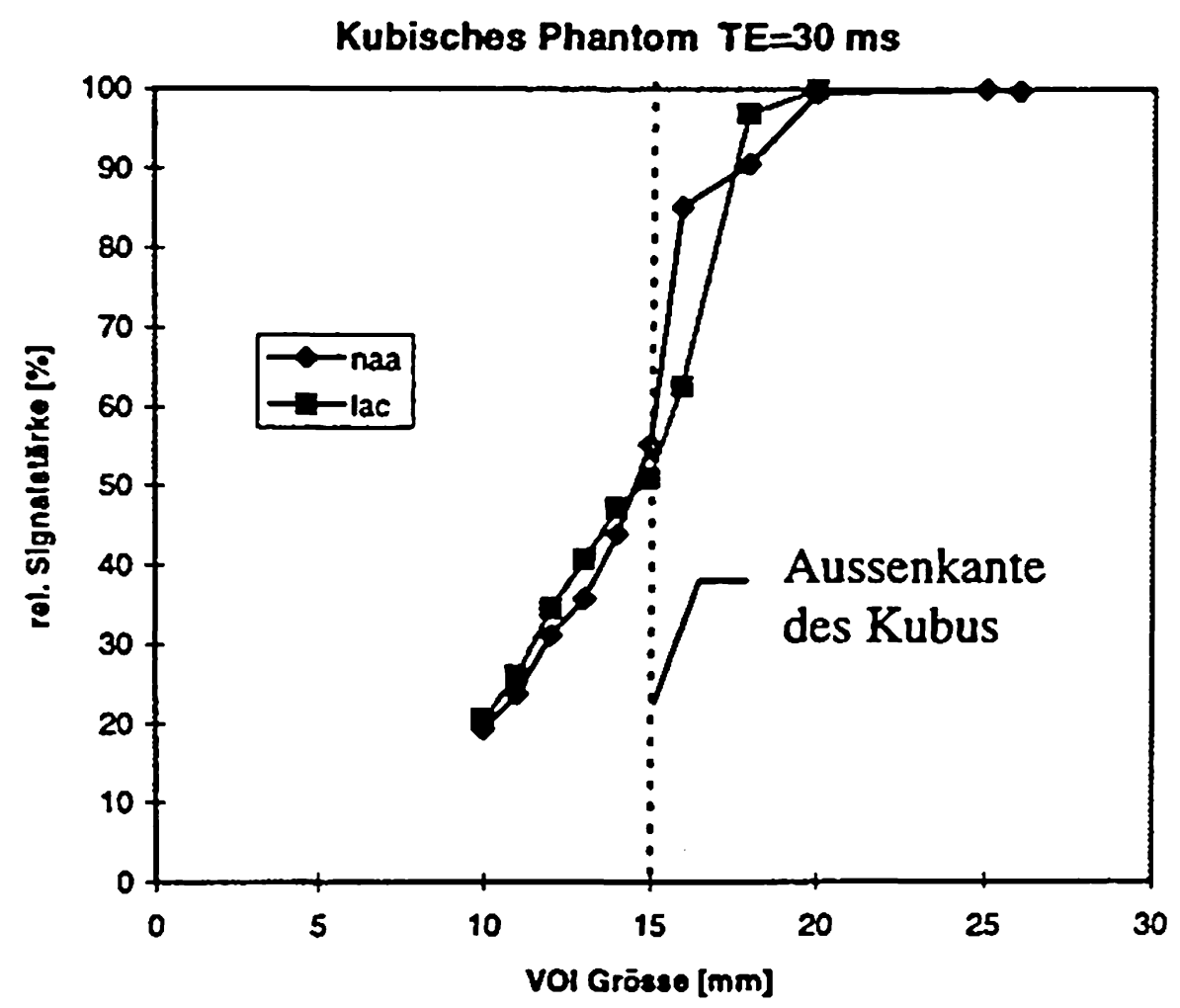

Bild 3: relative Signalstärke aufgetragen über Volumengrösse (VOI, Volume of interest) in der PRESSSequenz bei einer Echozeit $T E=30 \mathrm{~ms}$.

\section{ERGEBNISSE}

Für das Signal ungekoppelter Protonen ist die Effizienz im wesentlichen durch die Fraktion der Kernzone im Messvolumen gegeben. Die Kontamination von aussen kann mit einer, durch das Rauschen bedingten, oberen Schranke von $5 \%$ beziffert werden. Die Effizienz der Lokalisierung beträgt nur etwa $50 \%$, was in Übereinstimmung mit der Simulation steht (Bild 3).

Die Messungen zeigen, dass $95 \%$ des Signals von den Spins innerhalb des Nominalvolumens stammen, das nominale Messvolumen also wesentlich grösser als das Zielvolumen ist. Das bedeutet eine äusserst geringe Kontamination von aussen.

Für gekoppelte Spins sind die Signaleinflüsse durch die Volumenselektion komplizierter. Die breite Flipwinkelverteilung im Randbereich des Messvolumens hat verschiedene Effekte zur Folge, die gleichzeitig auftreten und deshalb schwer voneinander zu trennen sind. Der Polarisationstransfer sorgt für eine Modulation des Laktatsignals in Abhängigkeit von der Echozeit [2]. Dieser entsteht in den Bereichen, in denen der Flipwinkel vom Nominalwert $180^{\circ}$ abweicht. Die Signalschwankungen können in einer asymetrischen PRESSSequenz mit optimierten Sinc-Pulsen bis zu $10 \%$ ausmachen. Aufgrund der Volumenverschiebung durch den Chemical Shift treten im Randbereich des Volumens Gebiete auf, in denen die J-Kopplung nicht refokussiert wird [3]. Gegenüber ungekoppelten Spins treten Signalunterschiede in Abhängigkeit von der Gradientenstärke auf. Für kleine Volumina, also starke Gradienten, verschwindet der Signalunterschied, da hier die Volumenverschiebung durch den Chemical Shift gering ist.

\section{SCHLUSSFOLGERUNG}

Um eine geringe Kontamination zu erreichen, muss eine starke Abnahme der Lokalisierungseffizienz in Kauf genommen werden. In den Randbereichen des Messvolumens treten vor allem für gekoppelte Spins komplizierte Effekte im Spektrum auf. Die Effizienz der Lokalisierung und die Kontamination des Signals durch Spins ausserhalb des nominalen Messvolumens konnten experimentell in Übereinstimmung mit der Simulation bestimmt werden.

\section{LITERATUR}

[ 1] Th. Ernst, J. Hennig, D. Ott und H. Friedburg, NMR Biomed. 2:216-224; 1989

[2] M. Bunse, W. Jung. O. Lutz, K. Küper und G. Dietze, J. Magn. Reson. A 114:230-237;1995

[3] G. McKinnon und P. Bösiger, Magn. Reson. Med. Biol. IV:101-111;1990

[4] K. Prüssmann, Beitrag BMT 1996 